\title{
Renal dysfunction prevalence and clinical impact in heart failure
}

This article was published in the following Dove Press journal:

Research Reports in Clinical Cardiology

8 September 201 I

Number of times this article has been viewed

\author{
Alberto Palazzuoli \\ Susanna Benincasa \\ Stefanie Grothgar \\ Pasquale Di Sipio \\ Giovanni Paganini \\ Marco Pellegrini \\ Ranuccio Nuti \\ Department of Internal Medicine \\ and Metabolic Diseases, Cardiology \\ Section, Le Scotte Hospital, \\ University of Siena, Italy
}

\begin{abstract}
Chronic kidney disease (CKD) is associated with a significant increase in death and cardiovascular mortality. However the exact mechanism by which CKD impairs the cardiovascular outcome is not well established. Some reasons may lie in the association of CKD with several other cardiovascular and noncardiovascular disorders including accelerated systemic atherosclerosis, endothelial dysfunction, increased levels of inflammatory factors, anemic status, bone mineral dysfunction, electrolyte imbalance, and renin-angiotensin-aldosterone system (RAAS) activation. Therefore several risk factors such as hypertension, diabetes, lipid disorders, and older age are common in both conditions. In patients affected with heart failure (HF) a key role is represented by the neurohormonal activation. This condition causes fluid and sodium retention, peripheral vasoconstriction, as well as increased congestion and cardiac workload. Moreover, HF during the decompensated phases is often associated with a worsening renal function that leads to further RAAS activation, microvascular damage, and intrarenal flow redistribution. In order to clarify the interactions between these factors, several questions need to be answered: the universal definition of "worsening renal function," the identification of the best laboratory parameters to investigate renal function in terms of sensitivity and specificity, and a better definition of the comorbidities' role in the determination of the outcome, especially in patients with chronic HF. A clarification of these key points could lead to the individualization of new specific therapeutic targets and to a reduction in mortality and hospitalization in patients with HF and renal impairment.
\end{abstract}

Keywords: heart failure, outcome, renal insufficiency, worsening renal function

\section{Introduction}

Heart failure (HF) is the leading cause of hospital admissions to the Medicare population. In addition to its high prevalence, hospitalization for decompensated HF is associated with extraordinarily high rates of morbidity and mortality. The clinical presentation can depend on hemodynamic status, primary cardiac disorder, systemic pressure, and organ perfusion/damage. ${ }^{1}$

Among the damage to various organs, renal impairment has become increasingly recognized as an independent risk factor for morbidity and mortality. Chronic kidney disease (CKD) is an independent predictor of myocardial infarction (MI), stroke, and death among men and women, even in younger patients. It is well known that the progression of renal insufficiency (RI) leads to an increased risk of cardiovascular mortality that cannot be fully explained by conventional risk factors or older age. ${ }^{2}$ However several studies have reported a significant correlation between CKD or worsening renal function and impaired HF outcome.
Correspondence: Alberto Palazzuoli and Metabolic Diseases, Cardiology Section, S Maria alle Scotte Hospital, Viale Bracci 53100 , Siena, Italy Tel +39577585 363; +39577585 46I Fax+39577233480 Email palazzuoli2@unisi.it 
In recent European and American guidelines for HF management, renal dysfunction was considered an index of poor prognosis. ${ }^{3,4}$ Although in most common randomized clinical trials conducted in the HF field, patients with RI tend to be excluded despite the increasing recognition of the prevalence and importance of renal disease in patients with HF. There are many reasons to report renal function in the HF setting: the well-recognized clinical impact of RI in common cardiovascular diseases, the high prevalence of this condition in HF, and the need to know if the common treatment used in HF patients could be applied even in renal dysfunction as an associated condition.

Moreover, the way of evaluating renal function remains unspecified: many studies reported only baseline creatinine, as they reported at the same time other data such as age, sex, and weight, which would enable creatinine clearance or glomerular filtration rate (GFR) to be easily calculated. Despite these data, both these specific laboratory parameters are often not collected. Recently the term "worsening renal function" (WRF) has been regularly used to describe the acute and/or subacute changes that occur in kidney function after episodes of acute decompensated heart failure (ADHF). For all these reasons the use of an established consensus for the definition/classification of RI appears mandatory in clinical practice as well as in future studies enrolling HF patients. This would permit a better standardization in evaluating its incidence, temporal profile, and outcome across future epidemiologic investigations. On the basis of these criteria we described the prevalence and clinical impact of RI in more recent clinical trials in the setting of acute and chronic HF.

\section{Risk factors: implication in HF and renal dysfunction}

Classical and nonclassical risk factors have long been investigated as implicated in cardiac insufficiency and RI. The prevalence of cardiovascular morbidity and mortality is higher in patients with CKD than in the general population. Traditional risk factors associated with RI such as hypertension, diabetes, dyslipidemia, and microalbuminuria combine with other risk factors typically associated to RI: uremic state, vascular calcification, ventricular hypertrophy, impaired left ventricular (LV) myocardial relaxation and LV filling pressure are all factors that lead to accelerated atherosclerosis, coronary artery disease (CAD) and peripheral artery disease, congestive heart failure, and cardiac sudden death. ${ }^{5}$ In particular, hypertension, a major risk factor for $\mathrm{CAD}$, had a high prevalence in patients with CKD. At least $35 \%$ of patients with CKD have evidence of a prior ischemic event or process (angina or a history of MI). The prevalence of LV hypertrophy increases at each stage of CKD, reaching $75 \%$ at the time of dialysis initiation. ${ }^{6,7}$

Additional factors such as abnormal mineral metabolism, hyperparathyroidism, hyperhomocysteinemia, and an increase in pro-inflammatory cytokines have been associated with higher CV risk in RI. ${ }^{8}$ Disturbed calcium phosphate metabolism has been implicated in the development of vessel calcification. The pathophysiologic process is not completely understood; probably growth factor effects of secondary hyperparathyroidism and sclerosis of the major peripheral vessels cause increased afterload and subsequent LV dysfunction. ${ }^{9}$

Proceeding from a re-analysis of the Atherosclerosis Risk In Communities (ARIC) study, Muntner et al confirmed that the burden of classical risk factors, such as hypertension, diabetes, older age, and dyslipidemia, is substantially higher among patients with CKD than the general population. However, these factors vary considerably, depending on patient characteristics, including degree of RI and causes of kidney disease. ${ }^{10}$

In a subset analysis of the Third National Health and Nutrition Examination survey (NHANES III) registry, the same authors pointed out that patients with CKD had lower levels of apolipoprotein $\mathrm{A}_{1}$ and higher levels of homocysteine, lipoprotein A, fibrinogen and C-reactive protein (CRP). Furthermore they observed that patients who presented those higher levels of Apolipoprotein $\mathrm{A}_{1}$, homocysteine, lipoprotein A, fibrinogen, and CRP, but without evidence of CKD and CAD, higher were at higher risk to develop CHD. ${ }^{11}$ This observation underlines the importance of primary prevention, with lifestyle change and pharmacological treatment where indicated.

Even anemia should be considered a risk factor for the impairment of renal and heart function. Several studies in different patient populations found an association with anemia, impaired cardiac function, more health care utilization, and morbidity. From a pathophysiological standpoint, CHD can also cause renal failure due to a decreased cardiac output reducing renal perfusion (ie, forward failure); subsequent renal failure may lead to decreased endogenous erythropoietin levels and may ultimately induce anemia, leading to an increased cardiac workload, completing the vicious circle. Recently this has been called the "cardio-renal-anemia syndrome."12,13

\section{Renal dysfunction and HF outcome}

Data from several sources demonstrate that approximately $20 \%$ to $40 \%$ of patients admitted to a hospital for ADHF 
have an associated RI, defined on the basis of risk, injury, failure, loss of kidney function, and end-stage kidney disease (RIFLE) and Acute Kidney Injury Network (AKIN) criteria (Figure 1). ${ }^{14,15}$ The broad range in the reported incidence is largely attributable to the variability of the definitions of WRF, the differences in the observed time-at-risk, and the heterogeneity of the selected populations being studied. ${ }^{16,17}$

In the Prospective Outcomes Study in Heart failure (POSH) study, ${ }^{18}$ Cowie et al prospectively enrolled 248 patients with ADHF (ejection fraction $[\mathrm{EF}]<40 \%$ and dyspnea) across eight European countries (mean age 68, 74\% men). WRF was defined as an increase in serum creatinine $>26 \mathrm{mmol} / \mathrm{L}$ from in-hospital admission. The follow-up was completed in $95 \%$ of patients within 6 months. Nearly one-third of patients (29\%) developed WRF during hospitalization. The risk profile of this subgroup was characterized by a major prevalence of increased serum creatinine levels on admission, pulmonary edema, and a history of atrial fibrillation. Although the 30-day mortality and up to 6-month mortality of WRF patients did not increase significantly, their duration of hospital stay was 2 days longer. This was not confirmed in patients admitted for the first time for HF with preserved LV systolic function, where Rusinaru et $\mathrm{al}^{19}$ demonstrated that a low baseline estimated GFR (eGFR) is a potent predictor of long-term mortality. In this work, patients with RI at baseline who developed WRF during hospitalization had a particularly poor prognosis. In contrast with these findings, in the Evaluation Study of Congestive Heart Failure and Pulmonary Artery Catheterization Effectiveness (ESCAPE) trial researchers observed patients with advanced HF by hemodynamic monitoring using pulmonary artery catheter guidance and considered the following parameters: cardiac index, cardiac output, pulmonary capillary wedge pressure, and systemic vascular resistance. In these patients baseline RI results have demonstrated more clinical impact on the outcome than WRF, defined as a further creatinine increase of $>0.3 \mathrm{mg} / \mathrm{dL}$. The addition of hemodynamic monitoring to clinical assessment does not prevent WRF or improve renal function after discharge. ${ }^{20}$

In a retrospective analysis of 949 patients from the Outcomes of a Prospective Trial of Intravenous Milrinone for Exacerbations of Chronic Heart Failure (OPTIME-CHF), Klein et $\mathrm{al}^{21}$ investigated the relation between the admission values and changes in blood urea nitrogen (BUN) and eGFR, and the rate of death within 60 days after discharge. Independently of values at admission, an increase of $>10 \mathrm{mg} / \mathrm{dL}$ in BUN during the hospitalization was associated with a worse 60-day survival rate: BUN (per $5 \mathrm{mg} / \mathrm{dL}$ increase) had a hazard ratio (HR) of 1.08 (95\% confidence interval [CI] 1.01-1.16). The authors concluded that a higher admission BUN and increasing BUN during hospitalization, independently of admission values, are associated with a lower survival rate. Interestingly, BUN on admission and change in BUN during hospitalization were better predictors of poor outcome than the estimated GFR. Similar findings

\section{Acute kidney injury: RIFLE}

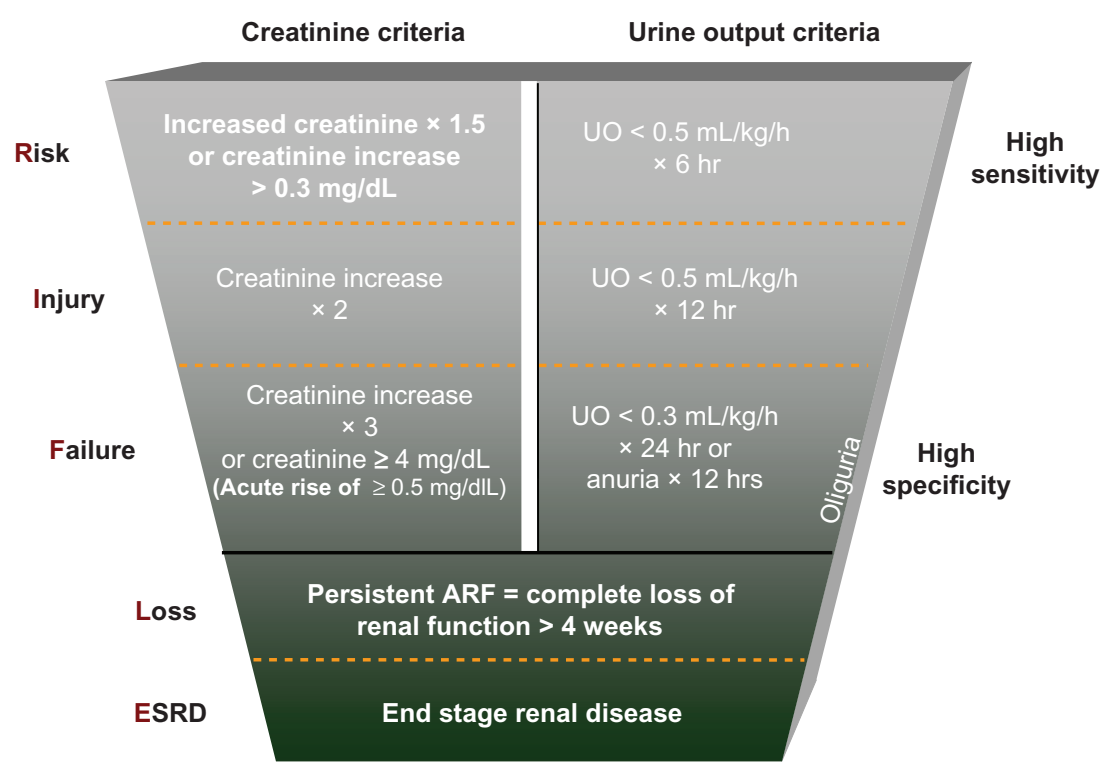

Figure I Acute kidney injury: risk, injury, failure, loss of kidney function, and end-stage kidney disease (RIFLE). 
were reported in a large Medicare cohort in which a higher BUN on hospital admission was a better predictor of postdischarge death than the creatinine-based measurements in patients hospitalized for MI or $\mathrm{HF}^{22}$

This observation could be explained by a higher baroreceptor-mediated nonosmotic arginine vasopressin (AVP) release which determines an increase in urea reabsorption in the collecting duct, leading to a BUN increase. In fact, in the OPTIME-CHF study, blood pressure levels were lower in the quartile of patients in whom BUN was higher. These patients would be expected to have higher baroreceptor-mediated nonosmotic AVP release. An increase in BUN was shown to be a marker of a more complex pattern of neurohormonal activation and not merely an index of renal dysfunction. ${ }^{23}$ In the Acute Decompensated Heart Failure National Registry (ADHERE), 30\% of hospitalized patients with HF had a history of chronic RI and $20 \%$ had a serum creatinine level $>2 \mathrm{mg} / \mathrm{dL}$ in an evaluation of 105,388 hospitalization episodes. ${ }^{24}$ The ESCAPE trial showed that $40 \%$ of advanced decompensated HF developed WRF during hospitalization in terms of creatinine clearance reduction, which is associated with an impaired outcome during the follow-up period. ${ }^{25}$

$\mathrm{RI}$ is a very common comorbidity also in patients affected by chronic HF. In a recent meta-analysis evaluating the impact of WRF in hospitalized HF patients during a 6-month follow-up, the authors showed a strong association with an unfavorable prognosis (odds ratio [OR] 1.62). ${ }^{26}$ Even in less advanced HF stages, RI demonstrated an association with increased LV systolic volume and a more advanced New York Heart Assocation (NYHA) class. Again, in the Studies Of Left Ventricular Dysfunction (SOLVD) trial, patients with moderate RI (creatinine clearance $<60 \mathrm{~mL} / \mathrm{min}$ ) experienced greater all cause mortality, pump failure death, and composite end point of death or hospitalization for worsening HF relative risk [RR] of $1.45 .{ }^{27}$ In a subsequent analysis of the same study, the authors found that in both the placebo and the enalapril group, older age, diuretic therapy, and diabetes were associated with decreased renal function, whereas a beta-blocker therapy and a higher ejection fraction were renoprotective. ${ }^{19}$ Older age was associated with a greater risk of developing decreased renal function in both groups, but significantly more so in the enalapril group. ${ }^{28}$ The Cooperative North Scandinavian Enalapril Survival Study (CONSESS) studied patients with severe chronic $\mathrm{HF}$ (CHF) and excluded patients if their serum creatinine was $>3.4 \mathrm{mg} / \mathrm{dL}$. While only about $10 \%$ of patients had a serum creatinine $>2.0 \mathrm{mg} / \mathrm{dL}^{29}$
In the Candesartan in Heart Failure-Assessment of Reduction in Mortality and Morbidity (CHARM) Study, Hillege et al found a high incidence of impaired GFR in all three groups studied independently of systolic function, and they showed that GFR reduction was a stronger predictor for mortality than LV ejection fraction (EF) or NYHA class. ${ }^{30}$ In the Valsartan in Heart Failure Trial (Val-HeFT), 5010 patients with class II, III, or IV heart failure were randomly assigned to receive valsartan or a placebo and were screened for $\mathrm{CKD}$ and proteinuria. At baseline, CKD was found in $58 \%$ and proteinuria in $8 \%$ of patients. Proteinuria was independently associated with mortality (HR 1.28, 95\% CI $1.01-1.62, P=0.05$ ) and first morbid event (HR 1.28, 95\% CI 1.06-1.55, $P=0.01)$. However, the increased risk of death associated with proteinuria was similar for those subjects with and without CKD (HR 1.26, 95\% CI 0.96-1.66 versus HR $1.37,95 \%$ CI $0.83-2.26 ; P=0.94)$, as well as for the first morbid event (HR 1.26, 95\% CI 1.01-1.57 versus HR 1.42, 95\% CI 0.98-2.07; $P=0.71) .{ }^{31}$

A recent systematic meta-analysis demonstrated that $29 \%$ of patients affected with CHF had moderate to severe RI, and that during a 1-year follow-up, mortality occurred in $38 \%$ and $51 \%$ of patients who, respectively, presented mild or severe RI compared with the patients with normal renal function. RI showed an incremental and linear increase in the mortality risk, with an increased risk of $15 \%$ for every $0.5 \mathrm{mg} / \mathrm{dL}$ of creatinine increase. ${ }^{32}$ In a retrospective analysis of data from 1129 patients, a discharge serum creatinine level $>2.5 \mathrm{mg} / \mathrm{dL}$ was the most powerful independent multivariate predictor of all-cause readmission (OR, 1.72). ${ }^{33}$ In a recent study, Tsagalis et al investigated the prevalence of $\mathrm{HF}$ and RI (defined as eGFR rate $<60 \mathrm{~mL} / \mathrm{min} / 1.73 \mathrm{~m}^{2}$ ) in patients with acute stroke: HF and RI were both independent predictors of mortality within 10 years and age, history of transient ischemic attacks and combined HF and RI were independent predictors of new cardiovascular events (Table 1). ${ }^{34}$

\section{Pathophysiologic mechanisms of $\mathrm{HF}$ in renal dysfunction}

The mechanisms by which the onset of acute HF or ADHF leads to AKI are multiple and complex. The pathophysiology of the acute renal injury during ADHF is poorly understood and likely involves interrelated hemodynamic and neurohormonal mechanisms which could contribute to the worsening of the cardiovascular outcome in these patients. Particularly, neurohormonal activation is more intense in patients with acute RI and it is associated with altered tubuloglomerular feedback. In addition, more recent studies have demonstrated 
the association between venous congestion rather than low cardiac output with WRF in ADHF. ${ }^{35}$ However, acute kidney injury leads to an increase in proarrhythmic risk, which could lead per se to an increased mortality. ${ }^{36}$ The clinical importance of each mechanism of acute renal injury is likely to vary from patient to patient (eg, acute cardiogenic shock versus hypertensive pulmonary edema). In acute HF, acute kidney injury (AKI) appears to be more severe in patients with impaired LV ejection fraction compared to those with preserved LV function, achieving an incidence $>70 \%$ in patients with cardiogenic shock. ${ }^{37}$

In chronic HF with concomitant RI other mechanisms have been proposed to explain the poor outcome. These include accelerated hypertension, LV hypertrophy, increased activation of the renin-angiotensin-aldosterone system (RAAS), reduced renal perfusion, diuretic resistance, and volume overload secondary to difficulties with sodium excretion. RAAS activation is predominant in patients with CHF and leads to several consequences: renal efferent vasoconstriction, increased peritubular capillary oncotic pressure, and reduced peritubular capillary hydrostatic pressure with kidney flow redistribution. Therefore, angiotensin itself causes proliferation of smooth muscle cells and adventitial fibroblasts in the vascular wall, intrarenal blood vessel thickening, intraglomerular hypertension, glomerulosclerosis, and tubulointerstitial fibrosis..$^{38,39}$ These hemodynamic consequences result in both fluid retention and sodium reabsorption with an increased central venous pressure, which, as previously reported ${ }^{35}$ is a key determinant of worsening renal function (Figure 1).

What is the relationship between common HF risk factors and WRF? When Forman et $\mathrm{al}^{40}$ analyzed WRF as a marker for poor outcome in patients with CHF or ADHF, the statistical analysis was adjusted for basal characteristics that may themselves determine WRF in patients with HF (male sex, kidney dysfunction at the time of hospital admission, worsened HF, tachyarrhythmia, and elevated blood pressure at hospital admission). In fact, these basal conditions can determine WRF and have also been demonstrated to be predictors of poor outcome in patients with HF, independently of their effect on renal function. Hillege et al in the CHARM study, ${ }^{30}$ showed how the number of comorbidities at baseline increased with a decreasing estimated GFR. In the study of Forman et al after adjusting for potential confounding factors including demographics (age, race), medical history (atrial fibrillation, cerebrovascular accident, HF, diabetes, use of digoxin), admission characteristics (orthopnea, hypotension, edema, high respiratory rate, systolic blood pressure $>160 \mathrm{mmHg}$ ), and lab values (potassium, creatinine, and BUN), the association between WRF and worse clinical outcomes remained significant. The authors proposed a model of four parameters (history of pre-existing HF, diabetes mellitus, admission creatinine of $1.5 \mathrm{mg} / \mathrm{dL}$ [ $132.6 \mu \mathrm{mol} / \mathrm{L}]$, admission systolic blood pressure $>160 \mathrm{mmHg}$ ) that are strongly and independently associated with WRF. This model distinguished the risks of developing WRF ranging from 10\% to $53 \%$ among different HF patients.

The Kidney Early Evaluation Program (KEEP), a multicenter screening program for volunteer participants at risk for CKD, demonstrated that in white Caucasians RI was better defined by the eGFR, while microalbuminuria was more prevalent in members of minority races. The authors suggested using both eGFR and spot albumin:creatinine ratio to identify RI, especially in members of minority racial and ethnic groups. ${ }^{41}$

On the basis of these data, specific studies to weigh the risk factors (including WRF) and to determine poor outcome in patients with HF are mandatory as well as studies to clarify the relationship between comorbidities and WRF.

\section{The concept of worsening renal function}

The above-cited data clearly highlight the common co-existence of heart and kidney dysfunction and their associated poor prognosis; however, some considerations are necessary: first, the definition of worsening renal function is not quite uniform in terms of cut off and method. As reported in the meta-analysis of Damman et $\mathrm{al}^{26}$ a rise in serum creatinine in patients with higher basal levels has a different meaning to a similar rise in patients with normal basal values. The authors concluded that the simplified Modification of Diet in Renal Disease (MDRD) formula is the most accurate to assess GFR in patients with $\mathrm{HF}$ and that mortality starts to increase significantly with an estimated GFR decrease of $>9 \mathrm{~mL} / \mathrm{min} / 1.73 \mathrm{~m}^{2}$. Many studies have validated estimated creatinine clearance (CockcroftGault) and eGFR from plasma creatinine concentrations (MDRD). But several methodological problems do exist: the patient population studied, the analysis method and the calibration of the creatinine method, the different GFR reference methods used, and the choice of the statistical method used for the comparison. MDRD is regarded as being less biased than GFR evaluated by Cockroft and Gault Formula; the creatinine-based formula is recommended for the follow-up of patients with CKD. ${ }^{42}$ However, a more accurate estimation of an individual renal function profile cannot be obtained only with a creatinine-based estimation of GFR. Even the evaluation 


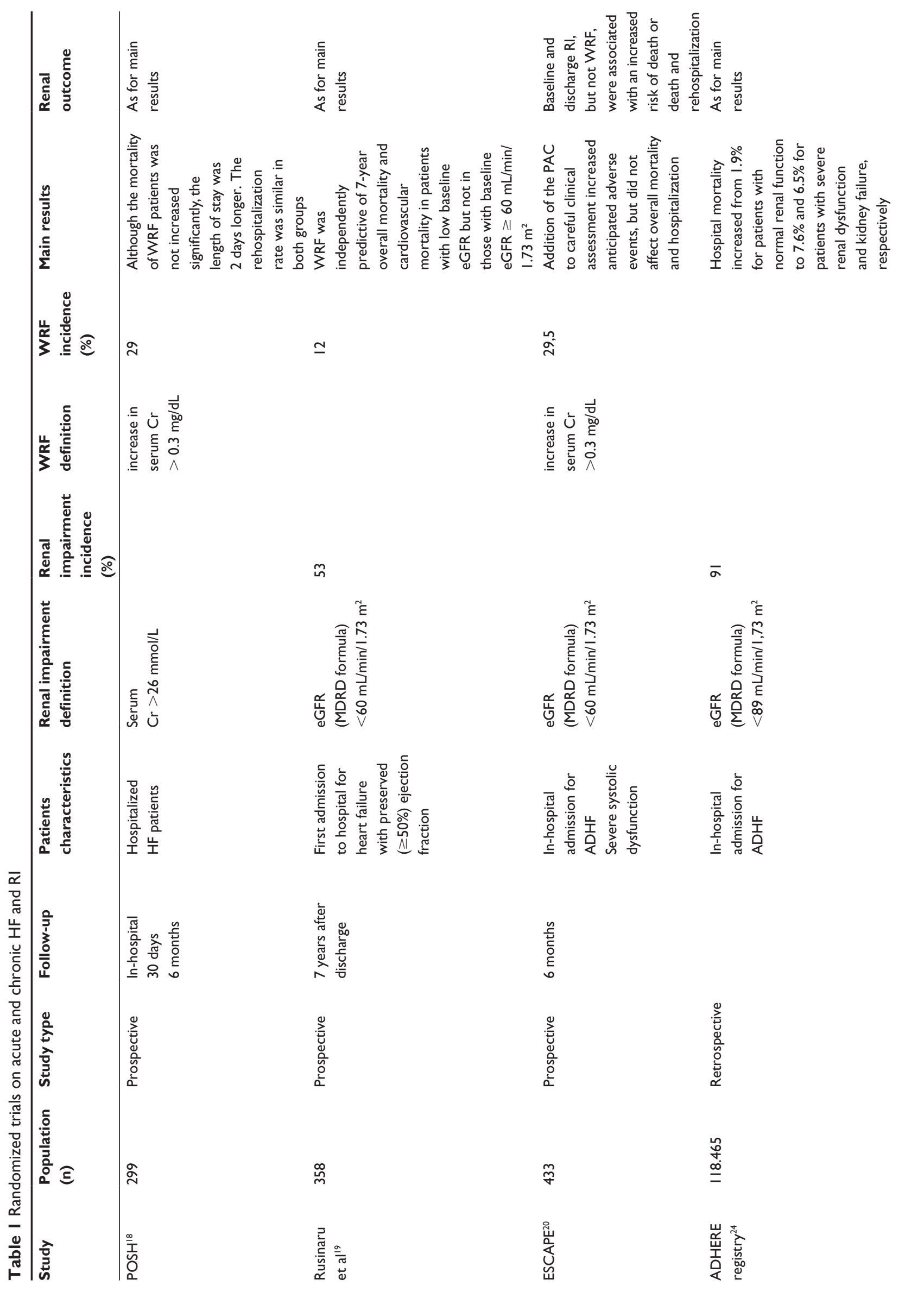




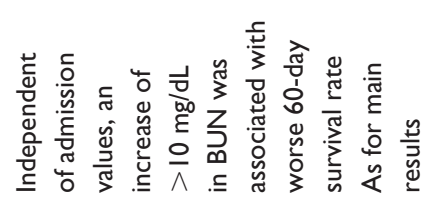
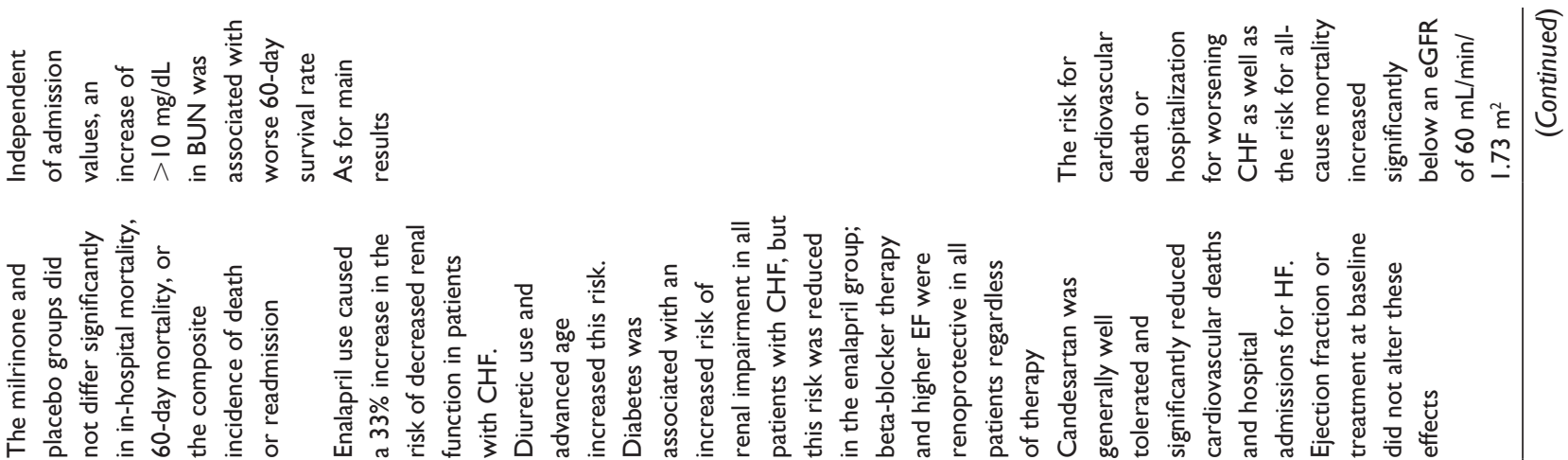

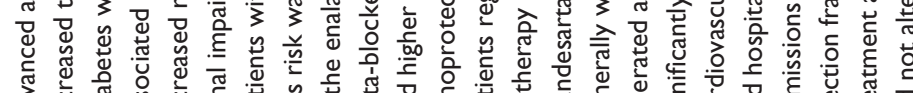

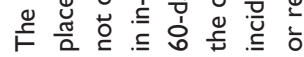

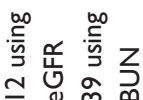

$\bar{m}$

离

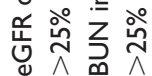

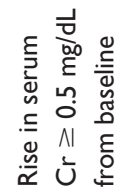

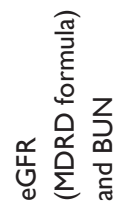

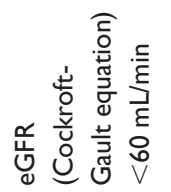

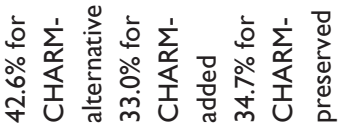

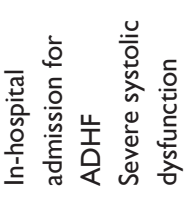

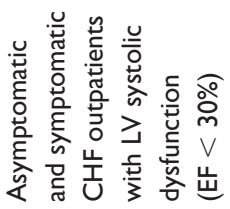

苁

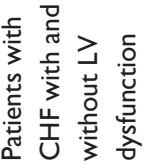

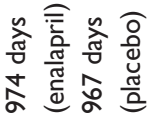

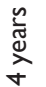

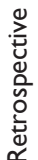

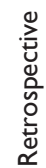

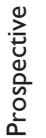

gे

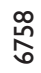

$\stackrel{\circ}{\sim}$

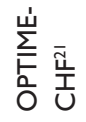

$\stackrel{\infty}{\text { Oे }}$

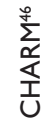




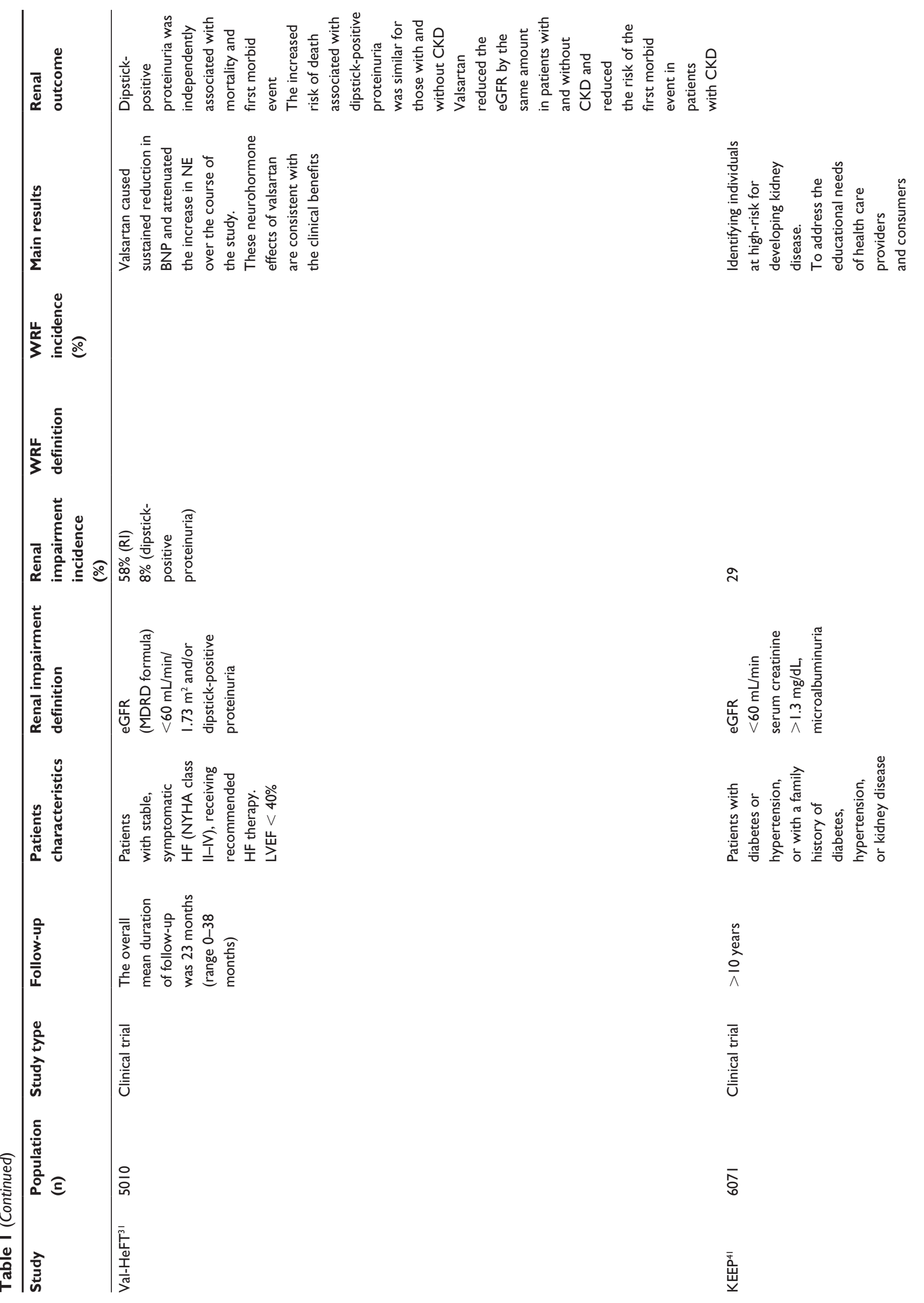




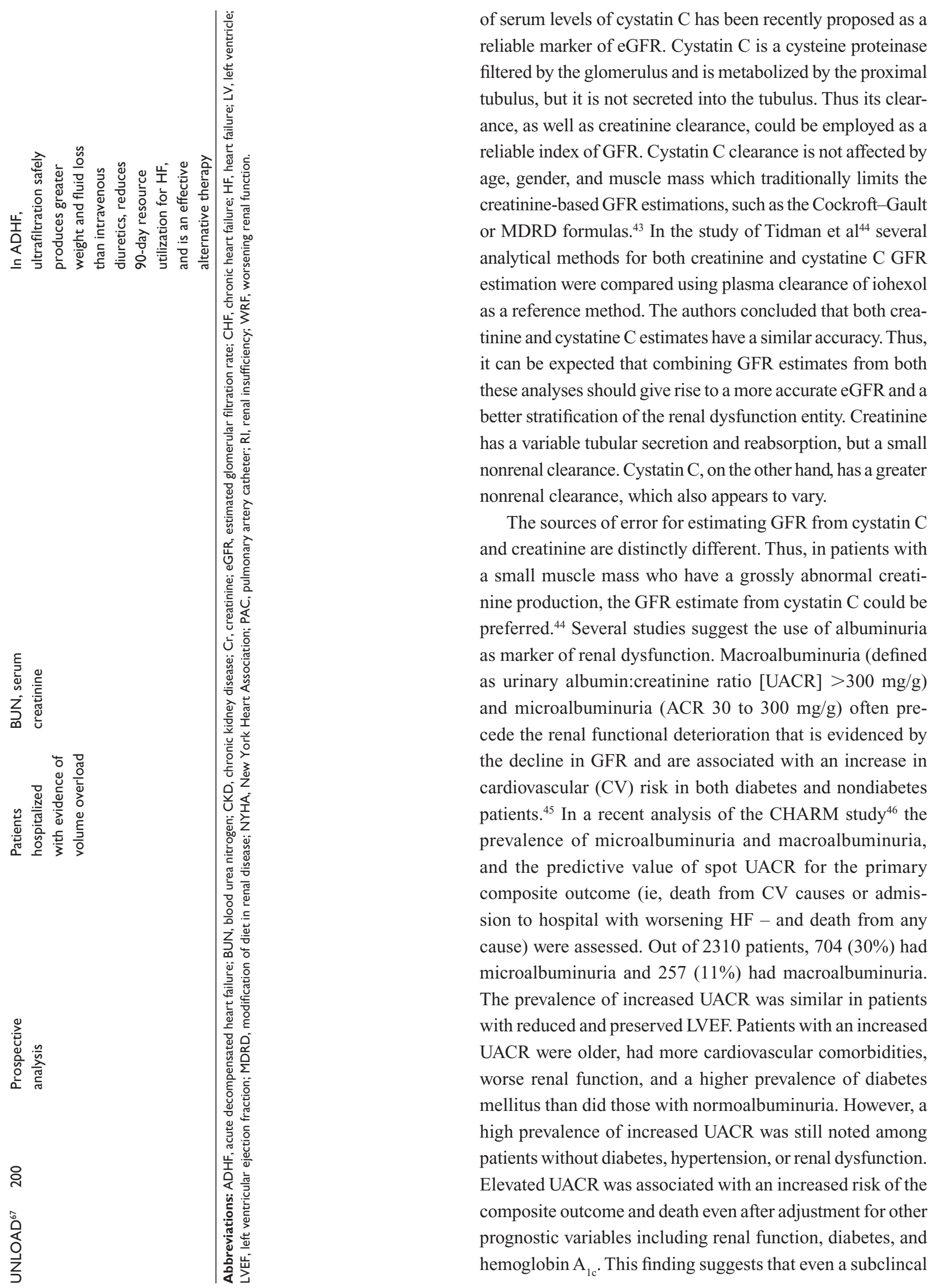


deterioration of renal function, as assessed by an albuminuria estimation, could have a significant negative impact on the outcome of patients with HF.

Several studies have also recently proposed the clinical use of serum neutrophil gelatinase-associated lipocalin (NGAL) levels in patients admitted to the hospital with ADHF, to estimate the risk of early worsening renal function. NGAL is produced by the nephron in response to tubular epithelial damage and is considered an early marker for acute renal tubular injury in several clinical settings. ${ }^{47,48}$ Recently, in 91 patients admitted to the hospital with ADHF Aghel et $\mathrm{al}^{49}$ observed significantly higher median admission serum NGAL levels in patients who developed WRF than in those without WRF (194 [interquartile range, IQR 150-292] $\mathrm{ng} / \mathrm{mL}$ versus 128 [IQR 97-214] ng/mL, $P=0.001$ ). They observed that patients with NGAL values $\geq 140 \mathrm{ng} / \mathrm{mL}$ at admission had a 7.4-fold increase in the risk of developing WRF, with a sensitivity and specificity of the cut-off of $86 \%$ and $54 \%$, respectively. There is no clear current consensus on a definition definition of WRF. (Table 2).

There is an urgent need to better analyze this aspect by specific studies. Some questions need to be clarified, in particular:

- What does the term WRF mean? What is the best laboratory index to define this condition? Can we obtain a universally recognized cut-off to define RI? Can we use the same cut-off for both patients with acute HF and CHF?

- Comorbidities in HF are determinants of poor outcome because they cause WRF: is it merely a marker of all these factors, or an independent contributor syndrome in the outcome of patients with HF?

Table 2 Protein biomarkers for early detection of acute kidney injury and heart failure

\begin{tabular}{ll}
\hline Biomarker & Associated injury \\
\hline KIM-I & $\begin{array}{l}\text { Ischemia and nephrotoxins } \\
\text { Ischemia and nephrotoxins } \\
\text { NGAL (lipocalin) }\end{array}$ \\
NHE3 & $\begin{array}{l}\text { Ischemia, prerenal postrenal AKI } \\
\text { Delayed graft function, } \\
\text { inflammatory activity } \\
\text { Myocardial injury, hemodynamic } \\
\text { Troponin T }\end{array}$ \\
Actin, actin depolymerizing factor & $\begin{array}{l}\text { Ischemia and delayed graft } \\
\text { function }\end{array}$ \\
BNP & $\begin{array}{l}\text { Hemodynamic overload, } \\
\text { neurohormonal activity } \\
\text { Hemodynamic overload, }\end{array}$ \\
NT-pro BNP & $\begin{array}{l}\text { neurohormonal activity } \\
\text { Proximal tubule injury }\end{array}$ \\
\hline
\end{tabular}

Abbreviations: BNP, brain natriuretic peptide; KIM-I, kidney injury molecule I; NGAL, neutrophil gelatinase associated lipocalin; NHE3, Na/K exchanger isoform.

\section{Therapeutic implications}

Patients with renal disease are more likely to die of CV disease than to progress to end-stage renal disease. ${ }^{50}$ Typically HF patients with creatinine $\geq 2.5 \mathrm{mg} / \mathrm{dL}$ have been systematically excluded from therapeutic trials, and therefore the optimal pharmacotherapy for patients with HF and RI remains uncertain. Coca et $\mathrm{al}^{51}$ reviewed 153 trials in order to quantify the representation of patients with renal disease in randomized controlled trials for interventions proven efficacious for $\mathrm{CV}$ disease. They revealed that 86 of 153 trials (56\%) excluded patients with renal disease and that absolute definitions (eg, threshold serum creatinine level) were used for renal disease in the protocols for only 5 trials. In most of the remaining cases, the criterion for exclusion of "renal disease" or the equivalent term was left up to interpretation by the individual site.

Current therapies for the majority comprise the use of inotropic and vasoactive agents, besides neurohormonal antagonists (ie, angiotensin-converting enzyme inhibitors [ACEi], AT1 receptor blockers, and beta-blockers) and diuretics. Only a minority of patients undergo mechanical support, ultrafiltration, or natriuretic peptide administration..$^{52}$

The rationale for effective treatment is to prevent the extracellular fluid overload by increasing the renal blood flow and inhibiting the RAAS pathways. ${ }^{53}$ The symptomatic benefit of diuretics in patients with HF has led to almost universal clinical acceptance, even though their efficacy and safety have never been evaluated in large randomized clinical trials. Use of diuretics involves a delicate balance. Although $\mathrm{HF}$ and RI frequently require increasing doses of diuretics, it is essential to carefully assess the therapeutic response to these drugs. The dose must be sufficient to achieve effective relief of fluid overload and its ensuing symptoms without stimulating adverse physiologic effects. ${ }^{54}$ The prevalence of diuretic resistance in the HF population is unknown. In a retrospective analysis of 1153 patients with advanced chronic HF, 402 patients had diuretic resistance (defined in this study as requirement of furosemide $>80 \mathrm{mg}$ or bumetanide $>2 \mathrm{mg}$ daily). Diuretic resistance was independently associated with total mortality, sudden death, and pump failure death. ${ }^{55}$

The use of inotropic agents is largely accepted, particularly in patients with low blood pressure and poor cardiac output, although clinical trials, despite an improvement in hemodynamic profile, were not able to demonstrate a clear benefit when administered: data from the ADHERE registry showed that patients receiving milrinone or dobutamine had significantly greater in-hospital mortality than those who received nitroglycerin or nesiritide.$^{56}$ In the ESCAPE trial, after adjustment for 
renal function and blood pressure, the use of inotropic agents was associated with a significantly worsened outcome. ${ }^{57}$ Physiologically, low-dose dopamine $(<5 \mu \mathrm{g} / \mathrm{kg} / \mathrm{min})$ may increase renal blood flow by direct vasodilatation (dopamine receptors), and at higher doses by increasing cardiac output (betareceptors) or increasing perfusion pressure via vasoconstriction (alpha-receptors). Dopamine also inhibits aldosterone release and inhibits sodium-potassium ATP-pump at the tubular epithelial cell level, resulting in increased sodium excretion and thereby diuresis. ${ }^{58,59}$

In spite of extensive use in clinical practice of low-dose dopamine in patients with or at risk for acute or WRF, there is insufficient evidence of real clinical benefits from this treatment. A large meta-analysis by Kellum and Decker, which included data from 51 studies published from 1966 to 2000 on the use of dopamine to prevent and/or treat renal dysfunction, showed that dopamine did not prevent mortality (RR 0.90 [0.44-1.83]; $P=0.92$ ), onset of acute renal failure (RR 0.81 [0.55-1.19]; $P=0.34$ ), or need for dialysis, (RR 0.83 [0.551.24]; $P=0.42$ ). The authors concluded that "the use of lowdose dopamine for the treatment or prevention of acute renal failure cannot be justified on the basis of available evidence and should be eliminated from routine clinical use." 60

Vasodilators decrease preload and afterload by reducing ventricular work, increasing stroke volume and cardiac output. ${ }^{61}$ Human B-type natriuretic peptide has multiple biologic and pharmacologic effects, and in its synthetic recombinant form, nesiritide, has been approved by the US Food and Drug Administration for the treatment of acute heart failure syndrome (AHFS). ${ }^{62}$ In the context of cardiorenal dysfunction, renal effects of nesiritide was first addressed by Wang et $\mathrm{al}^{63}$ who studied the effect of nesiritide versus placebo in 15 patients with CHF. The results showed that nesiritide had no effect on GFR, renal plasma flow, urine output, or sodium excretion. Sackner-Bernstein et al ${ }^{64}$ performed a meta-analysis of randomized, double-blind, parallel-group controlled trials of nesiritide (versus placebo or active control) in patients with ADHF to assess the risk of WRF. The results suggested that nesiritide may have adverse impacts on renal function. Despite these negative studies, subsequent studies and observations have suggested that nesiritide may still hold promise as a renal-protective therapy in advanced HF therapy when used in appropriate doses. The Follow-Up Serial Infusion of nesiritide trial, ${ }^{65}$ was designed to look at intermittent infusion of nesiritide in patients with comorbid advanced HF and RI. Infusions were given either once weekly or twice weekly over 12 weeks. Nesiritide at these two doses was well tolerated with no increase in incidence of WRF. Recently, Owan et al ${ }^{66}$ evaluated the effects of nesiritide at the standard dose of $2 \mu \mathrm{g} / \mathrm{kg} / \mathrm{min}$ followed by an infusion of $0.01 \mu \mathrm{g} / \mathrm{kg} / \mathrm{min}$ for 48 hours in 72 patients with AHFS and renal dysfunction: they did not reveal any renal function impairment in terms of creatinine and BUN increase. The role of nesiritide as a renal-protective and dieresis-promoting therapy in AHFS remains promising but requires further study.

In the Ultrafiltration Versus Intravenous Diuretics for Patients Hospitalized for Acute Decompensated Heart Failure (UNLOAD) trial, a prospective, randomized, multicenter trial of early ultrafiltration versus intravenous diuretics in 200 patients hospitalized with HF, mild RI, and hypervolemia, despite similar fluid loss with ultrafiltration and continuous diuretic infusion, at 90 days there were mild significant differences between the two groups in terms of patients rehospitalized for $\mathrm{HF}$ (16 of 89 [18\%] versus 28 of 87 [32\%]; $P=0.037$ ), and length of hospitalization (1.4 to 4.2 versus 3.8 to 8.5 in days; $P=0.022$ ). The only significant advantage in the ultrafiltration group was in terms of unscheduled office and emergency department visits (14 of 65 [21\%] versus 29 of 66 [44\%]; $P=0.009) .{ }^{67}$ In the most recent AHA practice guidelines, ${ }^{4}$ hemofiltration or dialysis were recommended in patients with a serum creatinine $>5 \mathrm{mg} / \mathrm{dL}$, to control fluid retention, minimize the risk of uremia, and to allow the patient to respond and to tolerate the drugs routinely used for the management of HF. In the OPTIME-CHF study, ${ }^{21}$ a significant rise in jugular venous pressure was observed, as quartile BUN values rose. The associated volume overload determines an activation of RAAS. In this clinical context, hemofiltration therapy, reducing fluid overload, could potentially reduce RAAS activation. ${ }^{23}$ In The Registry to Improve the Use of Evidence-Based Heart Failure Therapies in the Outpatient Setting (IMPROVE-HF) it was reported that, in patients with $\mathrm{HF}$ and LV dysfunction, the severity of concomitant chronic kidney disease was an independent predictor of adherence to ACEi/ARBs therapy, but not to any of the other guideline recommended measures in outpatient cardiology practices. ${ }^{68}$ The potential benefit of ACEi/angiotensin-receptor blocker (ARB) therapy in patients with HF and moderate to severe RI is still unclear. Masoudi et al reported similar or even increased survival rates for ACEi-treated HF patients with concomitant severe RI compared with patients with moderate IR or normal renal function. ${ }^{69}$ In contrast to these findings, Ezekowitz et $\mathrm{al}^{70}$ did not observe any potential benefit in terms of mortality rates in patients with ischemic $\mathrm{HF}$ and creatinine clearance $<30 \mathrm{~mL} / \mathrm{min}$ who underwent ACEi therapy. ${ }^{32}$ Two studies have probed the potential benefit of therapy with the beta-blocker carvedilol in dialysis patients with dilated cardiomyopathy. In a follow-up of this patient group over 
several years, there were superior survival and fewer hospitalizations in the beta-blocker-treated group compared with placebo, suggesting a potential beneficial role for carvedilol in this clinical setting. ${ }^{71,72}$ Recently a potential beneficial role in patients with ADHF and RI has been proposed for rolofylline, an adenosine $A_{1}$ receptor antagonist. The PROTECT trial $^{73}$ has been designed to investigate its potential role and to determine an efficacious dose. In 301 patients hospitalized for AHF with an estimated creatinine clearance of 20 to $80 \mathrm{~mL} / \mathrm{min}$ and elevated natriuretic peptide levels within 24 hours of presentation, placebo or rolofylline 10,20 , or $30 \mathrm{mg}$ was administered as 4-hour infusions for 3 days in addition to intravenously administered loop diuretics. Serum creatinine increased in patients receiving placebo and remained stable or tended to decrease in those receiving rolofylline. After 2 weeks of treatment a stabilization in the increase in creatinine levels was observed in patients who received rolofylline compared with those who received placebo; increased dose of rolofylline showed a linear correlation with the decrease of creatinine levels, although less significant $(r=-0.12, P=0.030)$. The authors also observed that treatment with rolofylline $30 \mathrm{mg}$ was associated with a trend toward reduced 60-day mortality or early readmission for CV or renal failure (HR 0.55; 95\% CI 0.28-1.04).

Hyponatremia can be considered a common occurrence in severe decompensated HF and a complication of diuretic therapy. The pathogenesis of this disorder could be due to a more severe activation of the RAAS, sympathetic nervous system, and higher AVP levels. Hyponatremia is often associated with serum volume expansion that necessitates an increase in the use of diuretics. In the OPTIMIZE-CHF registry, patients with hyponatremia had significantly higher in-hospital and follow-up mortality rates and longer hospital stays. This population showed increased risk of in-hospital mortality and follow-up mortality. ${ }^{74}$ Tolvaptan, a selective V2-receptor antagonist, was tested as a new drug in patients with ADHF. In ACTIV and CHF studies a trend toward lower mortality was found in the subgroup of patients with high levels of BUN or severe systemic congestion. A post hoc analysis confirmed BUN as significant predictor of both mortality and the composite endpoint of death or HF hospitalization at 60 days. However in terms of the clinical outcome, these data should be interpreted with caution: these were phase II studies, with a relatively small database, and perhaps all the factors that could have affected the outcome may not have been analyzed, which could have confused the association between BUN levels and outcome. In the Efficacy of Vasopressin Antagonism in Heart Failure Outcome
Study With Tolvaptan (EVEREST) short- and long-term effects of tolvaptan in patients hospitalized with ADHF and documented evidence of impaired LVEF were investigated. Although a significant clinical improvement (dyspnea, edema, body weight, and serum sodium) was revealed in the early phase, the longer-term safety outcome trial showed no effect, favorable or unfavorable, on its primary outcome. ${ }^{75}$ More targeted studies are needed to improve the therapeutic strategies in patients with RI and HF.

\section{Conclusion}

HF and RI are two clinical conditions that are often associated particularly in some subtypes of patients with common risk factors and CV characteristics (ie, hypertension, diabetes, older age, high atherosclerotic burden). RI in HF patients has become increasingly recognized as an independent risk factor for mortality, unfortunately the most important clinical trials in HF tend to exclude patients with RI. Moreover RI and HF may worsen each other by amplifying pathophysiologic mechanisms that lead to the development of a vicious circle. Because of several confounding factors, it remains still unclear whether a WRF specifically contributes to a poor outcome or whether it is merely a marker of advanced cardiac failure and RI. The lack of specific studies evaluating the clinical impact and strategic treatment of RI in acute $\mathrm{HF}$ and $\mathrm{CHF}$ means that a univocal clinical approach has not been established. Thus, the association between the two conditions needs to be further studied with multicentric clinical trials in this field.

\section{Disclosure}

The authors declare no conflicts of interest in relation to this paper.

\section{References}

1. McAlister FA, Lawson FM, Teo KK, et al. A systemic review of randomized trials of disease management programs in heart failure. $\mathrm{Am}$ J Med. 2001;110:378-384.

2. McCullough PA, Li S, Jurkovitz CT, et al; KEEP Investigators. Chronic kidney disease, prevalence of premature cardiovascular disease, and relationship to short-term mortality. Am Heart J. 2008;156:277-283.

3. Task Force for Diagnosis and Treatment of Acute and Chronic Heart Failure 2008 of European Society of Cardiology, Dickstein K, Cohen-Solal A, et al. ESC Guidelines for the diagnosis and treatment of acute and chronic heart failure 2008: the Task Force for the Diagnosis and Treatment of Acute and Chronic Heart Failure 2008 of the European Society of Cardiology. Developed in collaboration with the Heart Failure Association of the ESC (HFA) and endorsed by the European Society of Intensive Care Medicine (ESICM). Eur Heart J. 2008;29:2388-2442.

4. Heart Failure Society of America. HFSA 2006 comprehensive heart failure practice guideline. J Card Fail. 2006;12(1):e1-e2.

5. Drüeke TB, Massy ZA. Atherosclerosis in CKD: differences from the general population. Nat Rev Nephrol. 2010;6:723-735. 
6. Levin A, Djurdjev O, Barrett B, et al. Cardiovascular disease in patients with chronic kidney disease: getting to the heart of the matter. Am J Kidney Dis. 2001;38:1398-1407.

7. Levin A, Thompson CR, Ethier J, et al. Left ventricular mass index increase in early renal disease: impact of decline in hemoglobin. Am J Kidney Dis. 1999;34:125-134.

8. Levin A. Clinical epidemiology of cardiovascular disease in chronic kidney disease prior to dialysis. Semin Dial. 2003;16:101-105.

9. Fatica RA, Dennis VW. Cardiovascular mortality in chronic renal failure: hyperphosphatemia, coronary calcification, and the role of phosphate binders. Cleve Clin J Med. 2002;69(Suppl 3):S21-S27.

10. Muntner P, He J, Astor BC, Folsom AR, Coresh J. Traditional and nontraditional risk factors predict coronary heart disease in chronic kidney disease: results from the atherosclerosis risk in communities study. J Am Soc Nephrol. 2005;16:529-538.

11. Muntner P, Hamm LL, Kusek JW, Chen J, Whelton PK, He J. The prevalence of nontraditional risk factors for coronary heart disease in patients with chronic kidney disease. Ann Intern Med. 2004;140:9-17.

12. Groenveld HF, Januzzi JL, Damman K, et al. Anemia and mortality in heart failure patients a systematic review and meta-analysis. J Am Coll Cardiol. 2008;52:818-827.

13. De Silva R, Rigby AS, Witte KKA, et al. Anemia, renal dysfunction, and their interaction in patients with chronic heart failure. Am J Cardiol. 2006;98:391-398.

14. Srisawat N, Hoste EE, Kellum JA. Modern classification of acute kidney injury. Blood Purif. 2010;29:300-307.

15. Bagshaw SM, Cruz DN, Aspromonte N, et al; Acute Dialysis Quality Initiative (ADQI) Consensus Group. Epidemiology of cardio-renal syndromes: workgroup statements from the 7th ADQI Consensus Conference. Nephrol Dial Transplant. 2010;25:1406-1416.

16. Latchamsetty R, Fang J. Prognostic value of transient and sustained increase in in-hospital creatinine on outcomes of patients admitted with acute coronary syndromes. Am J Cardiol. 2007;99:939-942.

17. Logeart D, Tabet J. Transient worsening of renal function during hospitalization for acute heart failure alters outcome. Int J Cardiol. 2008; 127:228-232.

18. Cowie MR, Komajda M, Murray-Thomas T, Underwood J, Ticho B; POSH Investigators. Prevalence and impact of worsening renal function in patients hospitalized with decompensated heart failure: results of the prospective outcomes study in heart failure (POSH). Eur Heart J. 2006; 27:1216-1222.

19. Rusinaru D, Buiciuc O, Houpe D, Tribouilloy C. Renal function and long-term survival after hospital discharge in heart failure with preserved ejection fraction. Int J Cardiol. 2011;147:278-282.

20. Binanay C, Califf RM, Hasselblad V, et al; ESCAPE Investigators and ESCAPE Study Coordinators. Evaluation study of congestive heart failure and pulmonary artery catheterization effectiveness: the ESCAPE trial. JAMA. 2005;294:1625-1633.

21. Klein L, Massie BM, Leimberger JD, et al; OPTIME-CHF Investigators. Heart Failure Predicts Postdischarge Survival: results from the Outcomes of a Prospective Trial of Intravenous Milrinone for Exacerbations of Chronic Heart Failure (OPTIME-CHF). Circ Heart Fail. 2008;1:25-33.

22. Smith GL, Shlipak MG, Havranek EP, et al. Serum urea nitrogen, creatinine, and estimators of renal function: mortality in older patients with cardiovascular disease. Arch Intern Med. 2006;166:1134-1142.

23. Schrier RW. Blood urea nitrogen and serum creatinine: not married in heart failure. Circ Heart Fail. 2008;1:2-5.

24. Heywood JT, Fonarow GC. High prevalence of renal dysfunction and its impact on outcome in 118, 465 patients hospitalized with acute decompensated heart failure: a report from the ADHERE Database. J Card Fail. 2007;13:422-430.

25. Mullens W, Abrahams Z, Francis GS, et al. Importance of venous congestion for worsening renal function in advanced decompensated heart failure. J Am Coll Cardiol. 2009;53:589-596.

26. Damman K, Navis G, Voors AA, et al. Worsening renal function and prognosis in heart failure: a systematic review and meta-analysis. J Cardiac Fail. 2007;13:599-608.
27. Dries DL, Exner DV, Domanski MJ, et al. The prognostic implications of renal insufficiency in asymptomatic and symptomatic patients with left ventricular systolic dysfunction. J Am Coll Cardiol. 2000;35: 681-689.

28. Knight EL, Glynn RJ, McIntyre KM, Mogun H, Avorn J. Predictors of decreased renal function in patients with heart failure during angiotensin-converting enzyme inhibitor therapy: results from the studies of left ventricular dysfunction (SOLVD). Am Heart J. 1999; 138(5 Pt 1):849-855.

29. Effects of enalapril on mortality in severe congestive heart failure. Results of the Cooperative North Scandinavian Enalapril Survival Study (CONSENSUS). The CONSESS Trial Study Group. $N$ Engl J Med. 1987;316:1429-1435.

30. Hillege HL, Nitsch D, Pfeffer MA, et al. Renal function as a predictor of outcome in a broad spectrum of patients with heart failure. Circulation. 2006;113:671-678.

31. Anand IS, Bishu K, Rector TS, Ishani A, Kuskowski MA, Cohn JN. Proteinuria, chronic kidney disease, and the effect of an angiotensin receptor blocker in addition to an angiotensin-converting enzyme inhibitor in patients with moderate to severe heart failure. Circulation. 2009;120:1577-1584.

32. Smith GL, Lichtman JH, Bracken MB, et al. Renal impairment and outcomes in heart failure: systematic review and meta-analysis. $\mathrm{J} \mathrm{Am}$ Coll Cardiol. 2006;47:1987-1996.

33. Krumolz HM, Chen YT, Wang Y, Vaccarino V, Radford MJ, Horwitz RI. Predictors of readmission among elderly survivors of admission with heart failure. Am Heart J. 2000;139:72-77.

34. Tsagalis G, Bakirtzi N, Spengos K, et al. Long-term prognosis of combined chronic heart failure and chronic renal dysfunction after acute stroke. Eur J Heart Fail. 2010;12:849-854.

35. Mullens W, Abrahams Z, Francis GS, et al. Importance of venous congestion for worsening of renal function in advanced decompensated heart failure. J Am Coll Cardiol. 2009;53:589-596.

36. Liang KV, Williams AW, Greene EL, Redfield MM. Acute decompensated heart failure and the cardiorenal syndrome. Crit Care Med. 2008;36:S75-S88.

37. Ronco C, Haapio M, House AA, Anavekar N, Bellomo R. Cardiorenal syndrome. J Am Coll Cardiol. 2008;52:1527-1539.

38. Joles JA, Koomans HA. Causes and consequences of increased sympathetic activity in renal disease. Hypertension. 2004;43:699-706.

39. Ljungman S, Laragh JH, Cody RJ. Role of the kidney in congestive heart failure. Relationship of cardiac index to kidney function. Drugs. 1990;39(Suppl 4):10-21.

40. Forman DE, Butler J, Wang Y, et al. Incidence, predictors at admission, and impact of worsening renal function among patients hospitalized with heart failure. J Am Coll Cardiol. 2004;43:61-67.

41. McCullough PA, Vassalotti JA, Collins AJ, Chen SC, Bakris GL. National Kidney Foundation's Kidney Early Evaluation Program (KEEP) annual data report 2009: executive summary. Am J Kidney Dis. 2010;55(3 Suppl 2):S1-S3.

42. Lamb EJ, Tomson CR, Roderick PJ. Estimating kidney function in adults using formulae. Ann Clin Biochem. 2005;42:321-345.

43. Perkins BA, Nelson RG, Ostrander BE, et al. Detection of renal function decline in patients with diabetes and normal or elevated GFR by serial measurements of serum cystatin $\mathrm{C}$ concentration: results of a 4-year follow-up study. J Am Soc Nephrol. 2005;16:1404-1412.

44. Tidman M, Sjöström P, Jones I. A comparison of GFR estimating formulae based upon s-cystatin $\mathrm{C}$ and s-creatinine and a combination of the two. Nephrol Dial Transplant. 2008;23:154-160.

45. Schiffrin EL, Lipman ML, Mann JF. Chronic kidney disease: effects on the cardiovascular system. Circulation. 2007;116:85-97.

46. Jackson CE, Solomon SD, Gerstein HC, et al; CHARM Investigators and Committees. Albuminuria in chronic heart failure: prevalence and prognostic importance. Lancet. 2009;374:543-550.

47. Wagener G, Gubitosa G, Wang S, Borregaard N, Kim M, Lee HT. Urinary neutrophil gelatinase-associated lipocalin and acute kidney injury after cardiac surgery. Am J Kidney Dis. 2008;52:425-433. 
48. Yndestad A, Landrø L, Ueland T, et al. Increased systemic and myocardial expression of neutrophil gelatinase-associated lipocalin in clinical and experimental heart failure. Eur Heart J. 2009;30:1229-1236.

49. Aghel A, Shrestha K, Mullens W, Borowski A, Tang WH. Serum neutrophil gelatinase-associated lipocalin (NGAL) in predicting worsening renal function in acute decompensated heart failure. J Card Fail. 2010;16:49-54.

50. Foley RN, Murray AM, Li S, et al. Chronic kidney disease and the risk for cardiovascular disease, renal replacement, and death in the United States Medicare population, 1998 to 1999. J Am Soc Nephrol. 2005; 16:489-495.

51. Coca SG, Yusuf B, Shlipak MG, Garg AX, Parikh CR. Long-term risk of mortality and other adverse outcomes after acute kidney injury: a systematic review and meta-analysis. Am J Kidney Dis. 2009 June;53(6):961-973.

52. Tavazzi L, Maggioni AP, Lucci D, et al. Nationwide survey on acute heart failure in cardiology ward services in Italy. Eur Heart J. 2006;27: 1207-1215.

53. Cody RJ. Diuretics in the management of congestive heart failure. Cardiologia. 1998;43:25-34.

54. Francis GS, Siegel RM, Goldsmith SR, Olivari MT, Levine TB, Cohn JN. Acute vasoconstrictor response to intravenous furosemide in patients with chronic congestive heart failure: activation of the neurohumoral axis. Ann Intern Med. 1985;103:1-6.

55. Neuberg GW, Miller AB, O’Connor CM, et al. Diuretic resistance predicts mortality in patients with advanced heart failure. Am Heart J. 2002;144:31-38.

56. Abraham WT, Adams KF, Fonarow GC, et al; ADHERE Scientific Advisory Committee and Investigators and the ADHERE Study Group. In-hospital mortality in patients with acute decompensated heart failure treated with intravenous vasoactive medications: an analysis from the Acute Decompensated Heart Failure National Registry (ADHERE). J Am Coll Cardiol. 2005;46:57-64.

57. Elkayam U, Tasissa G, Binanay C, et al. Use and impact of inotropes and vasodilator therapy during heart failure. Am Heart J. 2007;153(1): 98-104.

58. O’Hara JF Jr. Low-dose "renal" dopamine. Anesthesiol Clin North America. 2000;18:835-851, ix.

59. Goldberg LI. Pharmacological bases for the use of dopamine and related drugs in the treatment of congestive heart failure. J Cardiovasc Pharmacol. 1989;14(Supp1 8):S21-S28.

60. Kellum JA, Decker J. Use of dopamine in acute renal failure: a metaanalysis. Crit Care Med. 2001;29:1526-1531.

61. Ferreira A, Bettencourt P, Dias P, et al. Neurohormonal activation, the renal dopaminergic system and sodium handling in patients with severe heart failure under vasodilator therapy. Clin Sci. 2001;100:557-566.

62. US Food and Drug Administration. Cardiovascular and renal drugs advisory committee meeting transcript May 25, 2002 (3749t2. rtf). Available from: http://www.fda.gov/ohrms/dockets/ac/01/ briefing/3749b2.htm. Accessed September 28, 2001.

63. Wang DJ, Dowling TC, Meadows D, et al. nesiritide does not improve renal function in patients with chronic heart failure and worsening serum creatinine. Circulation. 2004;110:1620-1625.
64. Sackner-Bernstein JD, Skopicki HA, Aaronson KD. Risk of worsening renal function with nesiritide in patients with acutely decompensated heart failure. Circulation. 2005;111:1487-1491.

65. Yancy CW, Krum H, Massie BM, et al. Safety and efficacy of outpatient nesiritide in patients with advanced heart failure. Results of the serial infusion of nesiritide (FUSION II) trial. Circ Heart Fail. 2008; 1:9-16.

66. Owan TE, Chen HH, Frantz RP, et al. The effects of nesiritide on renal function and diuretic responsiveness in acutely decompensated heart failure patients with renal dysfunction. J Card Fail. 2008 May; 14(4):267-275.

67. Costanzo MR, Saltzberg MT, Jessup M, Teerlink JR, Sobotka PA; Ultrafiltration Versus Intravenous Diuretics for Patients Hospitalized for Acute Decompensated Heart Failure (UNLOAD) Investigators. Ultrafiltration is associated with fewer rehospitalizations than continuous diuretic infusion in patients with decompensated heart failure: results from UNLOAD. J Card Fail. 2010;16:277-284.

68. Heywood JT, Fonarow GC, Yancy CW, et al. Influence of renal function on the use of guideline-recommended therapies for patients with heart failure. Am J Cardiol. 2010;105:1140-1146.

69. Masoudi FA, Rathore SS, Wang Y, et al. National patterns of use and effectiveness of angiotensin-converting enzyme inhibitors in older patients with heart failure and left ventricular systolic dysfunction. Circulation. 2004;110:724-731.

70. Ezekowitz JA, Hernandez AF, Starling RC, et al. Standardizing care for acute decompensated heart failure in a large megatrial: the approach for the Acute Studies of Clinical Effectiveness of Nesiritide in Subjects with Decompensated Heart Failure (ASCEND-HF). Am Heart J. 2009;157(2):219-228.

71. Cice G, Ferrara L, DiBenedetto A, et al. Dilated cardiomyopathy in dialysis patients - beneficial effects of carvedilol: a double-blind, placebo-controlled trial. J Am Coll Cardiol. 2001;37:407-411.

72. Cice G, Ferrara L, D'Andrea A, et al. Carvedilol increases two-year survival in dialysis patients with dilated cardiomyopathy: a prospective, placebo-controlled trial. J Am Coll Cardiol. 2003;41:1438-1444.

73. Cotter G, Dittrich HC, Weatherley BD, et al; PROTECT Steering Committee Investigators, and Coordinators. The protect pilot study: a randomized, placebo-controlled, dose-finding study of the adenosine A1 receptor antagonist rolofylline in patients with acute heart failure and renal impairment. J Card Fail. 2008;14:631-640.

74. Rosner MH. Hyponatremia in heart failure: the role of arginine vasopressin and diuretics. Cardiovasc Drugs Ther. 2009;23:307-315.

75. Gheorghiade M, Gattis WA, O'Connor CM, et al. Effects of tolvaptan, a vasopressin antagonist, in patients hospitalized with worsening heart failure: a randomized controlled trial. JAMA. 2004;291:1963-1971.

76. O'Connor CM, Miller AB, Blair JE, et al; Efficacy of Vasopressin Antagonism in Heart Failure Outcome Study with Tolvaptan (EVEREST) investigators. Causes of death and rehospitalization in patients hospitalized with worsening heart failure and reduced left ventricular ejection fraction: results from Efficacy of Vasopressin Antagonism in Heart Failure Outcome Study with Tolvaptan (EVEREST) program. Am Heart J. 2010;159:841-849.
Research Reports in Clinical Cardiology

\section{Publish your work in this journal}

Research Reports in Clinical Cardiology is an international, peerreviewed, open access journal publishing original research, reports, editorials, reviews and commentaries on all areas of cardiology in the clinic and laboratory. The manuscript management system is completely online and includes a very quick and fair peer-review system.

\section{Dovepress}

Visit http://www.dovepress.com/testimonials.php to read real quotes from published authors. 